\title{
Investigation of silver nanoparticles on titanium surface created by ion implantation technology
}

This article was published in the following Dove Press journal:

International Journal of Nanomedicine

\author{
István Lampé \\ Dezső Beke ${ }^{2}$ \\ Sándor Biri ${ }^{3}$ \\ István Csarnovics ${ }^{4}$ \\ Attila Csik ${ }^{3}$ \\ Zsuzsanna Dombrádi ${ }^{5}$ \\ Péter Hajdu ${ }^{3}$ \\ Viktória Hegedűs ${ }^{6}$ \\ Richárd Rácz ${ }^{3}$ \\ István Varga ${ }^{7}$ \\ Csaba Hegedüs' \\ 'Department of Biomaterials and \\ Prosthetic Dentistry, Faculty of Dentistry, \\ University of Debrecen, Debrecen, \\ Hungary; ${ }^{2}$ Department of Solid State \\ Physics, University of Debrecen, \\ Debrecen, Hungary; ${ }^{3}$ Hungarian \\ Academy of Sciences, Institute for \\ Nuclear Research, Debrecen, Hungary; \\ ${ }^{4}$ Department of Experimental Physics, \\ Faculty of Science and Technology, \\ University of Debrecen, Debrecen, \\ Hungary; ${ }^{5}$ Department of Medical \\ Microbiology, Faculty of Medicine, \\ University of Debrecen, Debrecen, \\ Hungary; ${ }^{6}$ Department of Orthodontics, \\ Faculty of Dentistry, University of \\ Debrecen, Debrecen, Hungary; \\ ${ }^{7}$ Department of Periodontology, Faculty \\ of Dentistry, University of Debrecen, \\ Debrecen, Hungary
}

Correspondence: Csaba Hegedüs Department of Biomaterials and Prosthetic Dentistry, Faculty of Dentistry, University of Debrecen, Nagyerdei krt.

98, Debrecen H-4032, Hungary

Tel +3652255308

Fax +365 2255208

Email hegedus.csaba.prof@dental.unideb.hu
Objectives: Using dental Ti implants has become a well-accepted and used method for replacing missing dentition. It has become evident that in many cases peri-implant inflammation develops. The objective was to create and evaluate the antibacterial effect of silver nanoparticle (Ag-NP) coated Ti surfaces that can help to prevent such processes if applied on the surface of dental implants.

Methods: Annealing I, Ag ion implantation by the beam of an Electron Cyclotron Resonance Ion Source (ECRIS), Ag Physical Vapor Deposition (PVD), Annealing II procedures were used, respectively, to create a safely anchored Ag-NP layer on $1 \times 1 \mathrm{~cm}^{2}$ Grade 2 titanium samples. The antibacterial effect was evaluated by culturing Staphylococcus aureus (ATCC 29213) on the surfaces of the samples for 8 hours, and comparing the results to that of glass as control and of pure titanium samples. Alamar Blue assay was carried out to check cytotoxicity.

Results: It was proved that silver nanoparticles were present on the treated surfaces. The average diameter of the particles was $58 \mathrm{~nm}$, with a $25 \mathrm{~nm}$ deviation and Gaussian distribution, the the filling factor was $25 \%$. Antibacterial evaluation revealed that the nanoparticle covered samples had an antibacterial effect of $64.6 \%$ that was statistically significant. Tests also proved that the nanoparticles are safely anchored to the titanium surface and are not cytotoxic.

Conclusion: Creating a silver nanoparticle layer can be an option to add antibacterial features to the implant surface and to help in the prevention of peri-implant inflammatory processes. Recent studies demonstrated that silver nanoparticles can induce pathology in mammal cells, thus safe fixation of the particles is essential to prevent them from getting into the circulation.

Keywords: implants, peri-implant inflammation, oxyde layer, cytocompatibility, antibacterial effect, physical vapor deposition, geometrical model

\section{Introduction}

Using titanium dental implants for restoring lost dentition has become an accepted widely used routine in everyday dental practice during the last decades. As the number of positioned implants has increased significantly, the awareness of certain risks affecting the lifespan of the restorations has come to be a focus of interest. Inflammation processes of the soft and hard tissues surrounding the implant can cause chronic inflammation, leading to the loss of the implant and, thus, loss of the whole restoration. According to a meta-analysis, Derks and Tomasi ${ }^{1}$ estimated weighted mean prevalence of peri-implant mucositis and periimplantitis to be $43 \%$ and $22 \%$, respectively. This statistically high level of inflammation processes has led to the development of different non-surgical 
and surgical treatment procedures with questionable results. This high level of inflammation turns attention to the prevention of the process. Besides strictly followed oral hygiene and follow-up procedure guidelines, it would be beneficial if the system itself had an intrinsic anti-inflammatory effect that is on alert constantly to prevent and fight inflammation. Silver has a wellknown antibacterial effect, being poisonous for bacteria. The antibacterial effect of silver ions is based on several factors, like deactivating enzymes, associating with and denaturing DNA, disturbing cell membrane transport. Recently, several authors investigated the antibacterial effect of different sized and shaped silver nanoparticles layered on a titanium surface using different methods, the effect of which is still not well understood. Wang et $\mathrm{al}^{2}$ demonstrated reactive oxygen species extra- and intracellularly of bacteria placed on a titanium surface covered by silver nanoparticles. The explanation for the development of reactive species was thought to be the micro-galvanic effect between silver and titanium.

A number of chemical and physical ways are known and used for silver modification of the titanium/titanium oxide surface. ${ }^{3-7}$

In most applications, the size distribution, density, adhesion, and depth profile of silver nanoparticles on the surface are important since these parameters determine the mechanical stability, antibacterial activity, optical characteristics, and light-sensitivity. The efficiency of their influence on the charge carrier generation, plasmon field distribution, and probably on the bioactivity depends on their size and other geometrical parameters. In the case of the combined method of physical vapor deposition (PVD) with electron cyclotron resonance (ECR), the geometrical parameters of the particles could be controlled by the initial layer thickness, implantation parameters, time and temperature of heat treatment, as had been was done for gold nanoparticles before. ${ }^{8}$

This paper focuses on a new technology to create silver nanoparticles on a titanium surface with defined particle size and distribution and on the effect of these nanostructures on bacteria. The surface of titanium samples was affected by complex physical route of Ag-NP formation: thin $\mathrm{Ag}$ layer deposition, ion implantation, and thermal annealing. The antibacterial feature of the surface was investigated against Staphylococcus aureus. The effect of the technology and the parameters of created nanostructures, their influence on the surface structure, and on the antibacterial process were investigated.
The results of investigations of Shi et al, ${ }^{9}$ Pacurari et al, ${ }^{10}$ Rahman et al, ${ }^{11}$ and Doudi and Setorki ${ }^{12}$ suggest that nanoparticles, including silver nanoparticles in the circulation can lead to pathology, for example to atherosclerosis, DNA damage, neurotoxicity, and hepatotoxicity. InkielewiczStepniak et a ${ }^{13}$ demonstrated increased cytotoxicity of fluoride on fibroblasts when silver nanoparticles were present.

These data indicate the importance of securing silver nanoparticles on a titanium surface to prevent them from getting into the circulation. The aim of this study is to evaluate a new fix nanoparticle creating method and its antibacterial effect.

We hypothesize that the Ag-Np Ti surface created using this new ion implantation technology has a significant antibacterial effect.

\section{Materials and methods}

To achieve the previously described titanium surface covered by anchored silver nanoparticles, the original titanium surface was modified in four stages:

1. Annealing I

2. Ag ion implantation by the beam of an Electron Cyclotron Resonance Ion Source (ECRIS)

3. Ag Physical Vapor Deposition (PVD)

4. Annealing II

The resulting surfaces were analyzed by physical analytical investigation methods. These analytical methods are as follows:

\section{Stopping and range of ions in matter (SRIM)}

Previously, silver ion implantation simulation has been achieved by SRIM computer code to estimate the penetration depth of ions to be implanted. ${ }^{14}$ SRIM is a compilation of software which can estimate numerous parameters of transport of ions in matter. In this work, to calculate the depth of stopping ions, the Ion Stopping and Range in Targets application was used, which can also manage complex multi-layer configurations. SRIM also contains an option of a quick calculation which creates ion stopping distributions and even tables of stopping powers. These additional functions helped to get further information about the silver ion stopping into the titanium dioxide layer.

\section{Secondary neutral mass spectrometry (SNMS)}

The SNMS is a unique thin film analysis method which uses a post-ionization technique. In this method the neutral 
atoms are sputtered from a sample surface with the help of the $\mathrm{Ar}, \mathrm{Kr}$, Ne, etc. plasma and detected after postionization. For sputtering and post-ionization processes it is used as an inductively coupled low pressure radio frequency discharge. It is a suitable technique to measure the chemical composition of almost any type of sample, because the flux of atoms sputtered from the sample is representative of the stoichiometry of the top-most layer.

To evaluate the thickness of $\mathrm{TiO}_{2}$ layers before and after annealing-I sputtering experiments were carried out in an INA-X type SNMS system produced by SPECS GmbH, Berlin. ${ }^{15}$ The INA-X device uses Electron Cyclotron Wave Resonance (ECWR) plasma as an ion source for both sputtering and post-ionization with sputtering energies of a few $100 \mathrm{eV}$. The SNMS is equipped with a quadrupole type mass spectrometer (Balzers QMA 410) up to 340 AMU for detecting elements. The bombarding energy of $\mathrm{Ar}^{+}$ions over the sample was $350 \mathrm{eV}$, with a plasma pressure of $1.5 \mathrm{mbar}$ and a current density of $\sim 1 \mathrm{~mA} / \mathrm{cm}^{2}$. A round-shaped area of samples with a diameter of $2 \mathrm{~mm}$ was sputtered through a Ta mask. The sputtering time was converted to depth by calibration of sputtering rates with a profilometer.

\section{Profilometer}

The roughness of the surface was measured by Ambios XP-I type profilometer (Ambios Technology, USA) operated in line scan mode. Linear-scan stage and the high precision optical flat grant punctual measurements on nanoscale. The lateral resolution is limited up to 60,000 data points per scan line. By measuring the vertical stylus displacement as a function of position, we were able to measure surface roughness in nanometer vertical resolution with a stylus load of $1 \mathrm{mg}$.

\section{Scanning electron microscope (SEM)}

The morphology of the radiated and sputtered surfaces was investigated by SEM (Hitachi S4300-CFE, Japan). This is a type of electron microscope that produces images of a sample by scanning the surface by a focused electron beam. The electrons interact with atoms on the surface, which results in various signals that contain information about the topography and composition of the surface. This technique is able to capture high resolution images with high depth of field. To get spatially resolved information on the formed particles, SEM images were taken. Nanoparticles were clearly visible on the titanium surface. These nanostructures are evaluated by a computer program based on National Instruments' Vision Assistant 2011 After microbiological evaluation, samples - that were treated by ultrasonic device - were examined by SEM the second time to check the presence of nanoparticles even after ultrasonic treatment. In addition to the morphological characteristics, several other properties of the test samples can be tested depending on the equipment installed (eg, elemental composition).

\section{Energy dispersive $\mathrm{X}$-ray spectrometry (EDS)}

The composition of the surface was investigated by Energy Dispersive Spectrometer (Bruker XFlash 6, Germany, installed in the SEM unit). EDS is an analytical technique to define the elemental analysis of the matter. By the reason of each element has a unique atomic structure allows a unique set of peaks on the electromagnetic emission spectra. To induce the emission of the characteristic $\mathrm{X}$-rays of the samples, $15 \mathrm{keV}$ acceleration voltage was applied during the measurements. The X-ray spectrum is detected by the silicon drift detector (SDD).

\section{Sample preparation}

10x10 mm sized Grade 2 Titanium plates (99.6 at\%, Grade 2, Spemet Co., Taipei, Taiwan) were used. The plates were mechanically polished to \#2000 grit level using a Struers LaboPol-35 polishing machine with Struers LaboForce-Mi device (Struers, Denmark), followed by a $3 \mu \mathrm{m}$ diamond to produce a mirror-like surface. The substrates were then cleaned in acetone three times for 10 minutes. each in an ultrasonic cleaning bath at room temperature, followed by rinsing with distilled water for 30 minutes and air drying.

Such Titanium plates were used as samples for Gr.1. These plates were used for the further stages of surface treatment. For Gr.2, silver nanoparticles were created by annealing I, ECRIS, PVD, and annealing II procedures, carried out as below.

\section{Method of creating Ag-NP on Ti surface}

One of the novelties of this work was the technology of preparation of silver nanoparticles on the titanium surface. This technique has already been used for the creation of silver and gold nanostructures on different substrates. ${ }^{8,16}$

\section{Stage I: Annealing I}

The thickness of the oxide layer on the prepared surface was measured by SNMS. In order to increase the thickness of the oxide layer for Ag-NP production, annealing was 
carried out. All samples were annealed at $550^{\circ} \mathrm{C}$ for 5 hours under normal atmospheric conditions.

\section{Stage II: Ion implantation}

A $14.3 \mathrm{GHz}$ Electron Cyclotron Resonance Ion Source (ECRIS) can produce a variety of charged ion beams from $\mathrm{H}, \mathrm{He}, \mathrm{CO}, \mathrm{CH}_{4}, \mathrm{~N}, \mathrm{O}, \mathrm{Si}, \mathrm{Ne}, \mathrm{Ar}, \mathrm{Kr}$, and $\mathrm{Xe}$ gases and $\mathrm{C}$, $\mathrm{C}_{60}, \mathrm{Ca}, \mathrm{Fe}, \mathrm{Ni}, \mathrm{Zn}, \mathrm{Ag}$, and $\mathrm{Au}$ ions from solids. ${ }^{17,18}$ In the present study the method for silver ion beam production was developed. The neutral silver atoms were evaporated by a commercial filament oven (Pantechnik, France). The oven head was placed $4 \mathrm{~cm}$ from the injection plate inside the plasma chamber and filled by $170 \mathrm{mg}$ of pure silver (99.999\%). The optimal operating temperature was found between 850 and $950^{\circ} \mathrm{C}$. Oxygen was used as the support gas. The composition of the beam was: Ag: 3.8\%, O: 18\%, others $\left(\mathrm{H}, \mathrm{C}, \mathrm{OH}, \mathrm{H}_{2} \mathrm{O}\right): 68 \%$. A typical silver ion beam spectra is shown in Figure 1.

The plasma was optimized for Ag. ${ }^{1+}$ For charge states $(\mathrm{Q}=1-4)$, silver beam currents between 0.02 and $0.5 \mathrm{e} \mu \mathrm{A}$ were obtained (Figure 2), with $\mathrm{q}_{\mathrm{av}}=1.4$ average charge state. The size of the ion beam was circular with a $50 \mathrm{~mm}$ diameter. The ion beam from the plasma was extracted by $2 \mathrm{kV}$, and in the arrangement of our setup all of the extracted components of the plasma reached and hit the samples. The implantation depth of the ions with charges from 1 up to 4 is different. Ti samples were implanted by $1.5 * 10^{16}$ ion/ $\mathrm{cm}^{2} \mathrm{Ag}$ dose.

\section{Stage III: Silver deposition}

After ion implantation, a $7-8 \mathrm{~nm}$ thick silver layer was deposited on the titanium sample. The thin layer was deposited by magnetron sputtering technique from a pure silver target. The working gas was Ar, while the deposition was done at $6 \mathrm{~mA}$ current for 12 seconds. Therefore, the deposition rate was $0.6 \mathrm{~nm} / \mathrm{s}$ while the substrate was at room temperature.

\section{Stage IV: Annealing II}

The samples were annealed at $550^{\circ} \mathrm{C}$ for 15 minutes at Ar:H atmosphere to form Ag-NP. As the $\mathrm{TiO}_{2}$ layer was rather thin, due to heat treatment a Ti-Ag phase could form and nanoparticles were not formed. To solve this problem, a thicker layer of $\mathrm{TiO}_{2}$ was created by thermal annealing (Annealing I). The thickness of the $\mathrm{TiO}_{2}$ layer after Annealing I was measured by SNMS. After annealing the thin silver layer and silver nanoparticles were formed in the same way as is presented above.

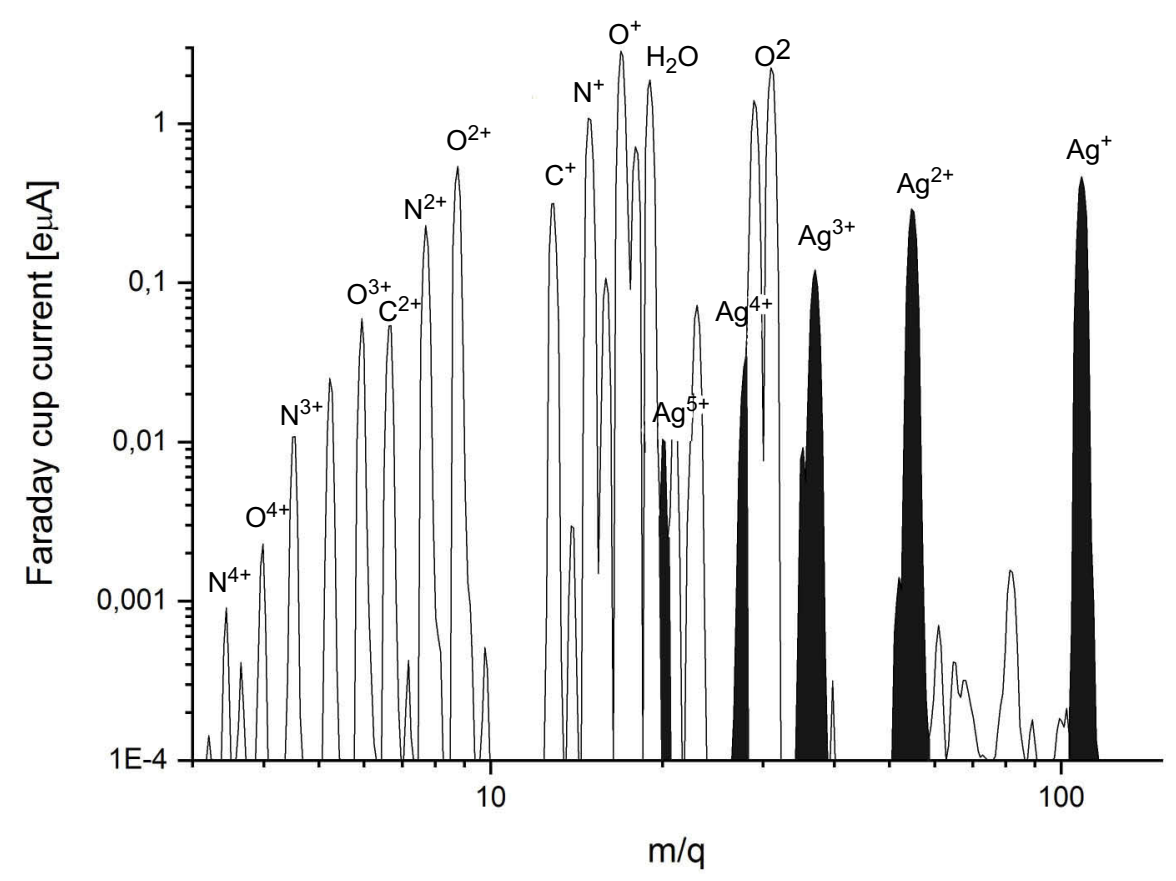

Figure I Silver ion beam spectra (optimized for $\mathrm{Ag}^{1+}$ ). 

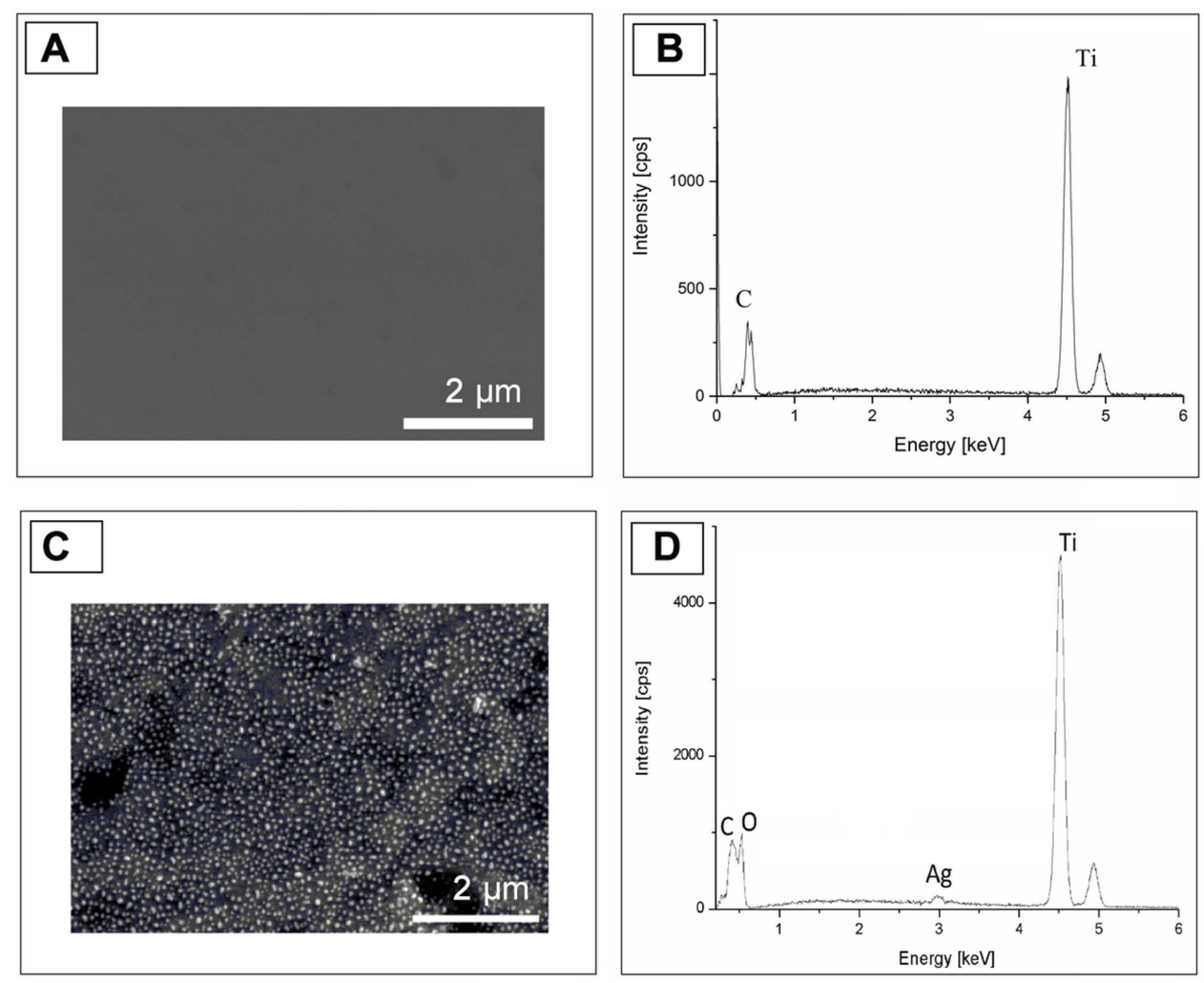

Figure 2 SEM image and EDS spectra of the surface before (A and B) and after (C and D) ECR+PVD treatment. Signal from the silver nanoparticle detected. Abbreviations: ECR, electron cyclotron resonance; EDS, energy dispersive X-ray spectrometry; PVD, physical vapor deposition; SEM, scanning electron microscope.

\section{Determination of antimicrobial efficiency}

The antimicrobial activity of each group of samples was tested with the gram-positive control strain Staphylococcus aureus (ATCC 29213) according to Wang et al. ${ }^{2}$ The bacterium was subcultured twice on Columbia blood agar overnight at $37^{\circ}$ C. A bacteria solution of $0.5 \mathrm{M}^{\mathrm{c}} \mathrm{Farland}$ density was prepared in brain heart infusion broth (BHI) and was diluted to a concentration of approximately $1 \times 10^{6}$ colony forming units $(\mathrm{CFU}) / \mathrm{mL}$.

The experiments were run in accordance with the ISO22196:2007(E) protocol. ${ }^{19}$ The $1 \mathrm{~cm}^{2}$ samples were placed on a glass microscope slide in a petri dish;50 $\mu \mathrm{L}$ $\left(0.05 \mathrm{~mL} / \mathrm{cm}^{2}\right)$ of the bacterium suspension were pipetted on the surface of each sample and subsequently covered with an aseptic borosilicate microscope cover glass carefully to prevent the leaking out of the suspension at the edges. ${ }^{20}$ Petri dishes were then incubated at $37^{\circ} \mathrm{C}$ in the dark.

After 8 hours of incubation, samples with cover slides were placed in $1 \mathrm{~mL}$ BHI broth, and the bacterial cells were detached from the surfaces by vigorous vortex mixing for 5 minutes followed by an ultrasonic disruption for 3 minutes. ${ }^{7,21}$ The bacterial suspensions were serially diluted in BHI broth for colony counting by the drop plate (DP) method of Herigstad et al, ${ }^{22}$ and $50 \mu \mathrm{L}$ of each suspension was dispensed in five evenly spaced $10 \mu \mathrm{L}$ drops on MuellerHinton agar. After the drops dried, the petri plates were inverted and incubated for 1 night at $37^{\circ} \mathrm{C}$, and the total count of CFUs per plate for the countable dilutions was recorded. Incubation was carried out in total darkness to prevent the photocatalytic effect of the titanium surface.

The efficacy of detachment was determined on control test pieces from each sample group that were inoculated with the bacterium suspension and were detached immediately without any incubation (zero time control). Each test was run in duplicate, and each set of experiments was repeated at least three times. Antibacterial rate was determined by the following formula: Antibacterial rate= (1-(CFU experimental group/CFU control group)) $\times 100 \%{ }^{2}$

Microscopic slide glass (10x10 mm sized) was used as a control surface.

Samples were used for evaluating the antibacterial effect after standard sterilization procedure in an autoclave.

Data of the antibacterial rates of two surfaces (titanium and silver-deposited titanium) were compared to the value 0 
corresponding to the control surface of glass by one-sample Student's $t$-test, whereas independent sample $t$-test was used to compare the two surface data. Further, the margin of errors for the mean estimations was also calculated. Note that, for the above methods, one needs to assume the normality of the antibacterial data (since it could only be tested with a fairly large sample otherwise).

\section{Cell viability assay}

The cell viability on the surfaces of different materials (glass, Ti, and Ag-Np implanted Ti) was examined by Alamar Blue $\operatorname{assay}^{\circledR}$ (Thermo Scientific, Waltham, MA, USA) for dental pulp stem cells (DPSCs). $4 \times 10^{4}$ cells were seeded to the surface of the investigated materials, then cultured for 14 days in DMEM F12 (Gibco, Waltham, MA, USA) supplemented with 10\% (v/v) fetal bovine serum (Gibco), 1\% Glutamax (Gibco), and 1\% Antibiotic-Antimycotic (Gibco). The culture medium was refreshed 3-times a week. Finally, the Alamar Blue assay was carried out according to the manufacturer's instructions. Briefly, the cell culture medium of each well was replaced with $10 \%$ (v/v) of Alamar Blue solution in each well, and after 1 hour incubation at $37^{\circ} \mathrm{C}$ in After 4 hours of incubation in a humidified atmosphere with $5 \% \mathrm{CO}_{2}$ at $37^{\circ} \mathrm{C}$, the fluorescence of samples $(100 \mu \mathrm{L})$ was measured using a microplate reader (HIDEX Sense Turku, Finland) at 544 nm excitation/ $595 \mathrm{~nm}$ emission.

\section{Scanning electron microscopy (SEM) examination of the dental pulp stem cells (DPSC) on the surface of the Ag-NP implanted $\mathrm{Ti}$}

DPSC cells on the material surface was evaluated by SEM, after the samples were fixed with $2 \%(\mathrm{v} / \mathrm{v})$ glutaraldehyde for 2 hours and with $1 \% \mathrm{OsO}_{4}$ for 1 hour, dehydrated using graded ethanol solutions $(10,30,50,70,80,90$, and $100 \%(\mathrm{v} / \mathrm{v}), 15$ minutes in each) and critical point dried using $\mathrm{CO}_{2}$. Finally, they were sputter-coated with gold for 30 seconds. The plasma current was $18-20 \mathrm{~mA}$, while the sputtering Ar pressure was 10-20 MPa during the coating. Samples were imaged using a scanning electron microscope (SEM, Hitachi S4300 CFE, Tokyo, Japan) at an accelerating voltage of $10 \mathrm{kV}$.

\section{Results}

\section{Sample surface characteristics}

The oxide layer increased on the titanium surface after Annealing I and it was investigated by SNMS. However, the roughness of the titanium surface influences the result of SNMS measurement. In our case the average roughness of the surface is so high as $R_{a}=8 \mathrm{~nm}$, which means there were several peaks and valleys on the nanoscale. Therefore, no sharp curves were observed on the SNMS depth profiles, thus only estimation of the thickness of the oxide layer was possible: it was about $25 \mathrm{~nm}$.

A SEM has been used to get 2D information of the surface configuration. Clear evidence was observed, nanoparticles were formed on the Ti surface as a result of annihilation after ion implantation and physical vapor deposition. Due to nanoparticles, the surface morphology has dramatically changed (Figures $2 \mathrm{~A}$ and $\mathrm{C}$ ). As the effect of treatment, the average roughness of the samples increased from $R_{a}=8 \mathrm{~nm}$ (with $R_{\max }=76 \mathrm{~nm}$ ) to $R_{a}=62 \mathrm{~nm}$ (with $R_{\max }=113 \mathrm{~nm}$ ). Element analysis of the surface by EDS built in the SEM proved that the structures formed on titanium surface are silver nanoparticles (Figure 2D).

SEM images were evaluated by a computer program based on National Instruments Vision Assistance. The results demonstrate that nanoparticles cover the surface with a $25 \%$ filling factor. The diameter distribution of nanoparticles follow Gaussian trend that is presented in Figure 3. The median is $58 \mathrm{~nm}$ with $25 \mathrm{~nm}$ deviation. The titanium peak is much higher than silver because the interaction volume of the EDS is on a micrometer-scale, while the size of silver nanoparticles is on a nanometer scale.

To check the physical stability of Ag-NP on the titanium surface of the samples that were used for antibacterial evaluation, thus ultrasonic treatment was carried out, were analyzed by SEM. SEM images were compared to samples with AG-NP where ECRIS was not applied. (In this case, Ag-NP-s were created by annealing I, PVD, and annealing II procedures applied.) It can be seen in Figures $4 \mathrm{C}$ and $\mathrm{D}$ that ultrasonic treatment released the silver nanoparticles from the surface when the PVD method was applied only. Detailed SEM investigations showed that the peeling of the oxide layer was responsible for the removal of the Ag particles. In Figure 4E, a border between the free surfaces (remained after peeling off) and the surface still covered by the remaining oxide layer with $\mathrm{Ag}$ nanoparticles is shown. EDS spectra revealed that the oxygen content was considerably different in the above two areas (Figure 4F). The filling factor changed from $\sim 25 \%$ to $\sim 1 \%$, as shown in Figures $4 \mathrm{~B}$ and D. For samples of Gr.2 (ECR+PVD), the ultrasonic treatment proved the high physical stability of the particles on the treated 


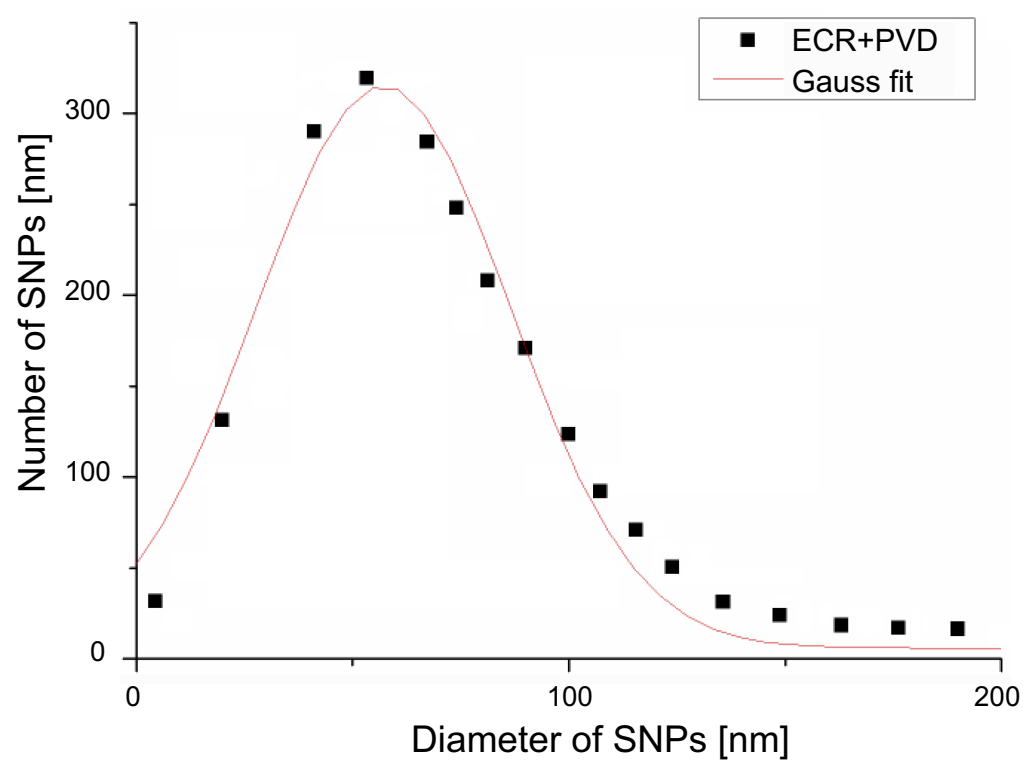

Figure 3 Density of the distribution of the diameter of silver nanoparticles (SNPs). Abbreviations: ECR, Electron Cyclotron Resonance; PVD, Physical Vapor Deposition.

surface (see Figures $4 \mathrm{~A}$ and $\mathrm{B}$ ). Several small areas in Figure 4B (see the arrows), however, indicate a similar peeling of the oxide layer with Ag particles, but the relative area of these are much less than for samples without ECR treatment. In this case the distribution has changed within the margin of error. One of the possible reasons is that the implanted silver ions are fixed onto the surface by treatment with ECR ion source. It is worth noting that ultrasonic treatment is much more drastic physical irritation when compared to conditions of normal tissue.

\section{Antibacterial effect}

The antibacterial properties of the prepared surfaces were evaluated with $S$. aureus, a common Grampositive bacterium. After culturing the bacterial cells for 8 hours on each sample the bacteria were collected and re-cultivated for colony counting according to the DP method. Figure 5 shows the antibacterial rates (\%) for each sample tested. Samples of Gr.1 had practically no effect on bacterial growth compared to the inert control glass surface ( $P$-value $>0.8$, the margin of error of the mean estimate is also rather large, $27.1 \%$ ). Inhibition of bacterial growth was unambiguously more robust in the case of Ag-NP containing surfaces. Our results demonstrate that the antibacterial effect is reproducible, and the inhibition is significant in comparison to the inert glass control and to the ineffective Ti surfaces. Gr.2 samples showed a mean of antibacterial rate of $64.6 \%$, with a margin of error of
$19.6 \%$ (with confidence level of $90 \%$ ) and a standard error of mean of $6.7 \%$, and the effect was statistically significant in Ag-NP sample in comparison to the control glass surface $(P$-value $=0.010)$, and also significant in comparison to the Ti surface $(P$-value $=0.005)$.

\section{Cell viability assay}

While antibacterial properties of the implanted materials are a very useful phenomenon, the used material and/or coating strategies should not have cytotoxic effects, especially as they will be used in the long-term. The cell viabilities on the different material surfaces are shown in Figure 6. As a control, the cells were seeded onto a glass surface, and untreated titanium surface was used to detect cytocompatibility of the implanted Ag nanoparticles. After 14 days both untreated $\mathrm{Ti}$ and $\mathrm{Ag}-\mathrm{Np}$ implanted Ti surface showed unchanged cell viability, compared to the glass surface control. These results revealed that implanted Ag$\mathrm{Np}$ has good biocompatibility for the long-term use, which is a favorable property when be used in dentistry or other areas of medicine. An SEM image of dental pulp stem cell (DPSC) on Ag-Np implanted $\mathrm{Ti}$ surface is shown in Figure 7.

\section{Discussion}

Using osseointegrated dental implants to restore lost dentition became a widely accepted and routinely used method in modern prosthetic dentistry. As the number of placed implants increases at a high rate from the early 1990s, 

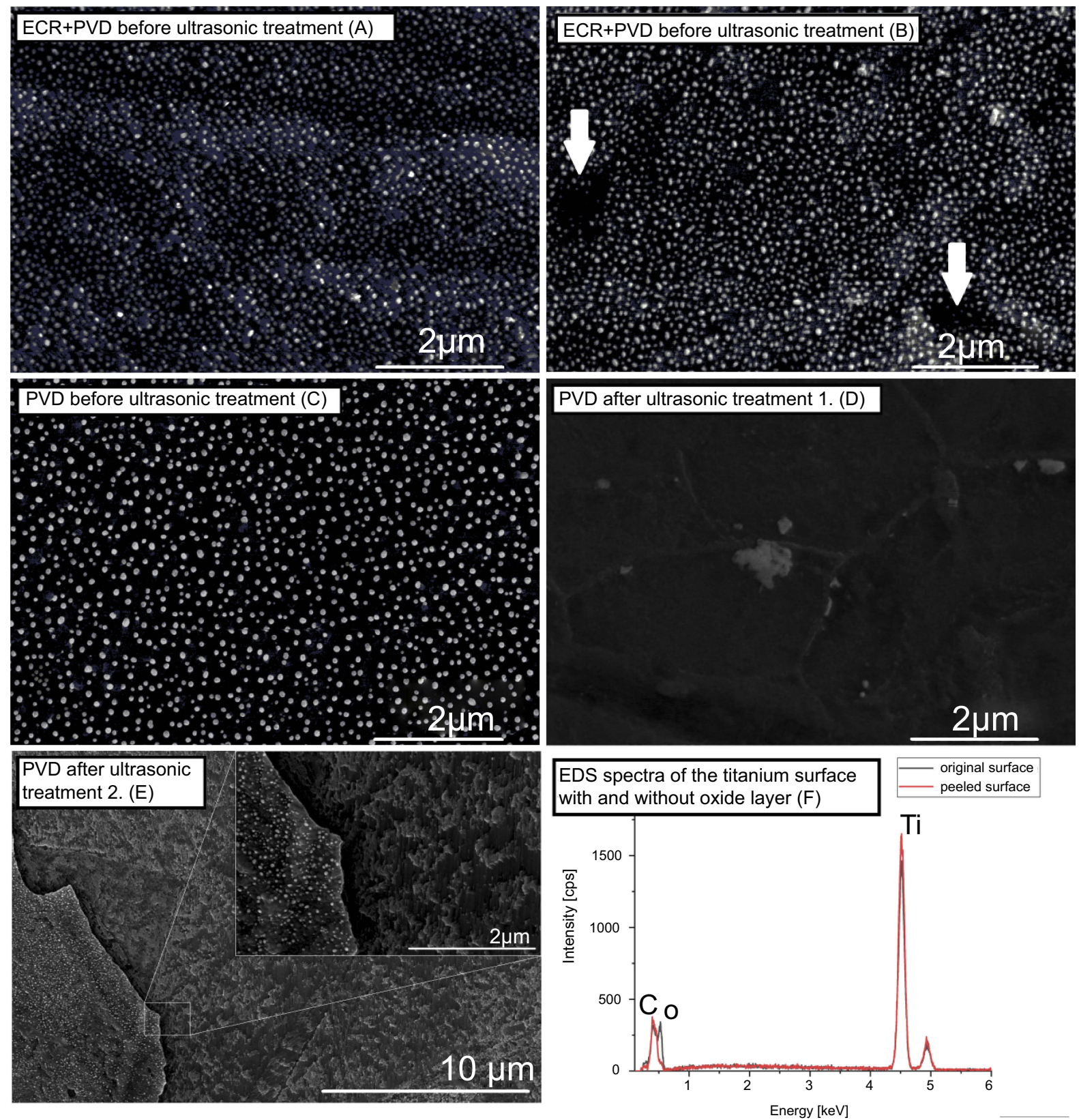

Figure 4 Demonstration of the effect of ultrasonic treatment to the nanoparticles on the surface in the case of ECR+PVD surface compared to samples where the surface was modified only by PVD method. (C and D) ultrasonic treatment released the nanoparticles from the surface when the PVD method was applied only. (A and B) with ECR+PVD only slight changes occurred (arrow). (E) Detailed investigation showed that peeling of the oxide layer was responsible for the removal of the Ag-Np-s. Peeling also occurred with ECR+PVD method (arrows on b), but the area is significantly lower than with PVD only. (F) oxygen content of the peeled off and the unpeeled areas are considerably different, indicating the removal of the $\mathrm{TiO}_{2}$ layer.

Abbreviations: ECR, electron cyclotron resonance; PVD, physical vapor deposition.

certain complications have arisen with the threat of losing the previously placed implant. Lindhe and Meyle ${ }^{23}$ presented the data of their working group at The European Workshop of Periodontology in 2008 that peri-implant inflammation was found in many patients having dental implants. Peri-implant mucositis was found in more than $50 \%$ of patients with implants, while peri-implantitis varied between $28 \%$ and $56 \%$. Several factors can be in the background, such as poorly designed or fabricated restoration, overload, bad oral hygiene or poor hygienic access, or surgical mistakes during placement. Systemic diseases or drugs can increase the risk for peri-implant inflammation. According to the guidelines of the European Association of Dental Implantologists at the 


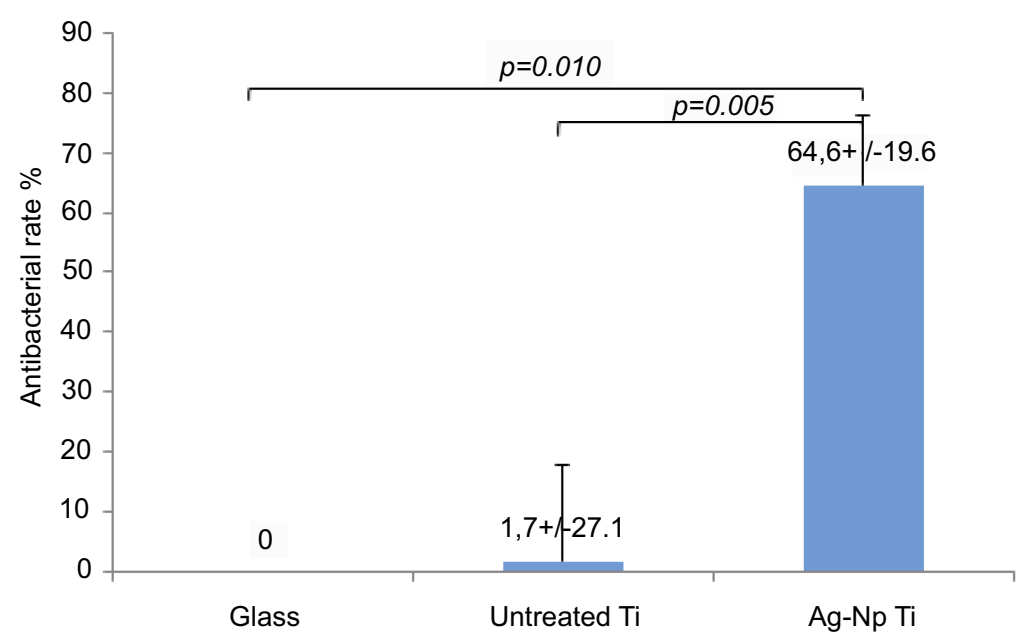

Figure 5 The bars represent the antibacterial rates of titanium and silver-deposited titanium surfaces with indicated standard deviation compared to control glass surface. The mean of three independent measurements in each case together with the margin of error for confidence level $90 \%$ are shown in each column (mean \pm margin of error).

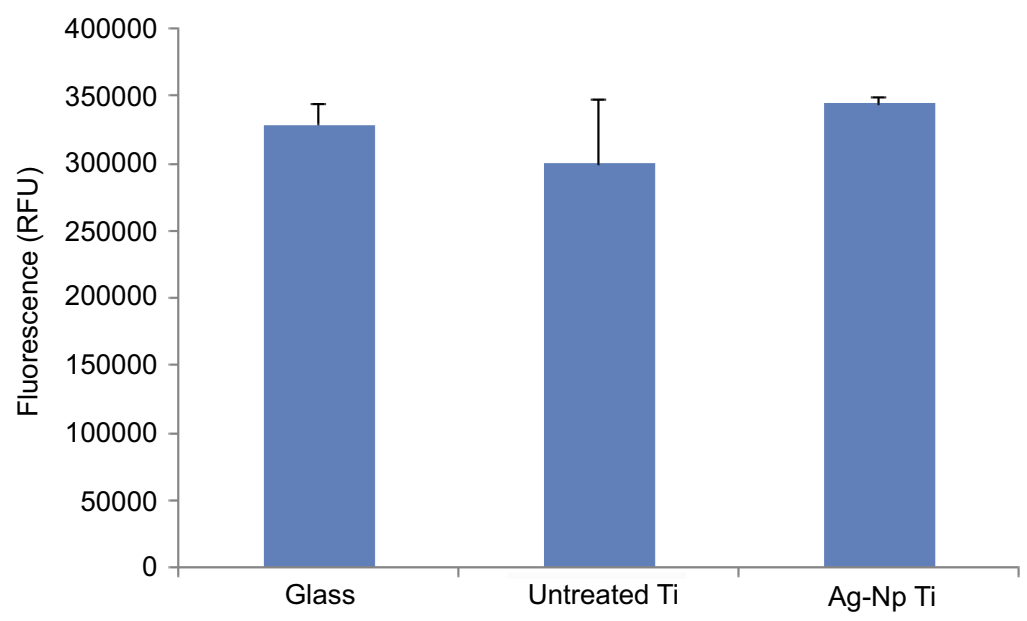

Figure 6 Alamar Blue assay of cell viability on different surfaces (glass, untreated Ti, Ag-Np implanted Ti) for I4 days ( $\mathrm{n}=3$ ).

10th European Consensus Conference (EuCC) 2015 in Cologne three forms of peri-implant inflammation can be classified: $^{24}$

1. Mucositis is the initial form being reversible and showing the signs of gingival inflammation

2. Peri-implantitis is a more advanced condition with loss of bone

3. Retrograde peri-implantitis in patients with unhealed periapical inflammation or periapical pathology due to burn of the bone during surgery.

During treatment, the risk factors should be eliminated, additionally bacteria are to be evacuated and a healthy environment is to be re-established. Treatment includes application of antibiotics and antibacterial agents, augmentation, and other surgical techniques. As with other diseases prevention should be taken to the first place to prevent the development of the pathology. As the prevalence of peri-implant inflammations is shown to be high, it would be advantageous if the implants themselves had antibacterial features.

Silver has been well known for its antibacterial effect for thousands of years. For its effect, it is becoming more and more widely used both in community health projects and individual antibacterial treatment procedures. Although the exact pathway of the effect still needs to be investigated, it is agreed that it has multiple points of attack. Silver ions alter the functions of the bacterial cell wall and membrane. Silver ions bond to sulphur and can block the function of proteins and enzymes containing sulphuric groups. Silver ions bond to DNA, blocking the replication process. ${ }^{25}$ Zheng et $\mathrm{al}^{21}$ found 


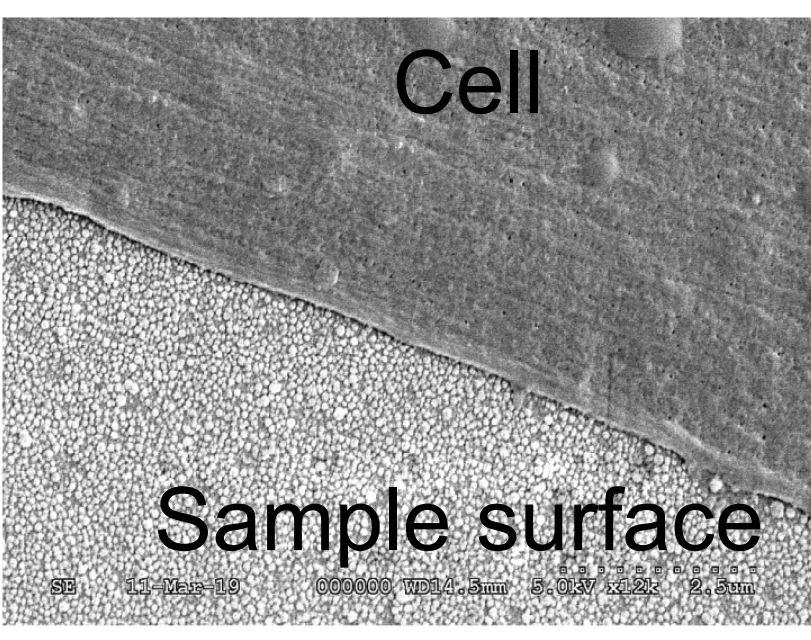

Figure 7 SEM image of DPSC on Ag-Np implanted Ti surface.

Abbreviations: DPSC, dental pulp stem cell; SEM, scanning electron microscope.

that gene expression of glucan-binding protein B in Str. mutans, fimbria protein A in Porphyromonas gingivalis, and agglutinin-like sequence4 in $C$. albicans is significantly reduced on the Ag-NP surface, confirming its antimicrobial effect. Glucan-binding protein B has an important role in cell wall construction, while fimbria protein A in Porpyromonas gingivalis and agglutinin-like sequence 4 in $C$. albicans play key roles in adhesion. Reducing expression of these genes results in an antibacterial effect.

Klueh et $\mathrm{al}^{26}$ suggested several mechanisms for $\mathrm{Ag}$ ions to become antibacterial:

- reaction with sulfhydryl group containing enzymes in the cell wall, transmembrane energy, and electrolyte transport mechanisms are altered

- blocking cytochrome-oxidase and NADH-succinatedehydrogenase to alter the respiratory mechanisms of the cell

- binding with bacterial DNA, causing denaturation by altering the bond between purines and pyrimidines

Zare and Shabani ${ }^{27}$ described phenomena that can result in bacterial death:

- interruption of ATP production and DNA duplication by the uptake of Ag ions

- disturbing of bacterial protein synthesis by Ag ions interacting with bacterial enzymes

- straight damage to cell membrane by Ag interacting with the peptidoglycan wall and the plasmatic membrane
Akhavan and Ghaderi ${ }^{28}$ found that the direct contact of the bacteria with sharp nanoparticles can cause mechanical alteration and disruption of the bacterial wall and cell membrane.

Wang et $\mathrm{al}^{2}$ proved that Ag-NP on the Ti complex has an antibacterial effect due to the micro-galvanic effect originated electron transfer between $\mathrm{Ti}$ and Ag-Np-s, and the induction of intracellular reactive oxygen species.

Prakash et $\mathrm{al}^{29}$ reported that a nanocomposite layer combined of $\mathrm{Ag}$ and $\mathrm{TiO}_{2}$ has a synergistic effect on antibacterial activity that is stronger than the antibacterial effect of $\mathrm{Ag}$ and $\mathrm{TiO}_{2}$ separately.

According to Liu

et $\mathrm{al}^{30}{ }^{30}$ the mechanism of this synergistic effect is the promoted Ag ion release and the increased surface energy by $\mathrm{TiO}_{2}$.

Mao et $\mathrm{al}^{31}$ mentioned the damage of the bacterial wall and the intracellular structure on the penetration of $\mathrm{Ag}$ and $\mathrm{Zn}$ ions into the bacterial cell. Jin et $\mathrm{al}^{32}$ and Mao et $\mathrm{al}^{31}$ also mentioned silver ions to stimulate the immune system by the production of a huge number of leokocytes and neutrophyls.

Silver used in nanoparticle form has a significantly higher surface that contributes to its much more effective antibacterial effect. On the other hand, nanoparticles are too small to be eliminated by the immune system, and they have the potential to cause toxic or inflammatory reactions. $^{33}$ Several studies have indicated that silver nanoparticles are cytotoxic to several cell lines. ${ }^{34}$ Shi et $\mathrm{al}^{9}$ demonstrated that Ag nanoparticles may cause injury and dysfunction of human endothelial cells, thus being hazardous for atherosclerosis. Inkielewicz-Stepniak et $\mathrm{al}^{13}$ found that exposure of human gingival fibroblasts to Ag$\mathrm{Np}$-s resulted in increased cellular damage effecting the cell membrane and mitochondria. Kang et $\mathrm{al}^{35}$ demonstrated that intracellular Ag-NP-s can result in cell death by the induction of production of reactive oxygen species, DNA damage, or compromised mitochondrial functions. Papers indicate Ag-NP-s causing apoptosis, by generating ROS production. ${ }^{36}$

Ag nanoparticles can be produced on titanium surfaces several ways. The technology used in the current experiment ensured a stable anchor for the Ag nanoparticles on the titanium surface. Ag ion implantation by ECRIS technique followed by annealing produced a surface configuration of stable Ag nanoparticles with a $58 \mathrm{~nm}$ average diameter and $25 \%$ filling factor. The colonization of the surface by $S$. aureus was investigated 
in the current study. The results showed a significant antibacterial effect of the generated surface on S. aureus compared to the native Ti surface or to the control glass surface. We have proved that all four steps of the combined treatment are required to reach the highest column in Figure 5, with physically stable Ag-NP-s on the antibacterial surface.

In our experiment we managed to create a titanium surface covered by silver nanoparticles with a proven significant antibacterial effect. The diameter distribution of nanoparticles follow a Gaussian trend; the median is 58 $\mathrm{nm}$, with a $25 \mathrm{~nm}$ deviation. The filling effect was $25 \%$. Figure 2 (left) shows a SEM picture of the surface with the anchored nanoparticles, while Figure 3 presents the related Gaussian curve.

Cao et $\mathrm{al}^{37}$ reported that Ag-NP-s of bigger size (5-25 $\mathrm{nm}$ in diameter) had a superior antibacterial effect than smaller (4 $\mathrm{nm}$ in diameter) ones. The technology used ensured safely anchored nanoparticles on the titanium surface, their role in the presented antibacterial effect is not relevant. It is only the released $\mathrm{Ag}$ ions that generated the effect.

Since the antibacterial effect of silver is known, we think that the larger surface of silver coming into contact with bacteria intensify the antibacterial effect of the surface. One can explain the effect of silver nanoparticles by the increase of the antibacterial surface. In order to see what would be the optimal geometrical arrangement of the $\mathrm{Ag}$ nanoparticles, the following geometrical consideration was carried out.

Our geometrical model treats nanoparticles as hemispheres; the surface and volume are counted as follows:

The thickness of the deposited continuous silver film, $d$, is given by

$$
d=V_{\text {Ago }} / A_{h}
$$

where $V_{A g o}$ is the volume of the film, and $A_{h}$ is the surface of the substrate covered. The effective surface of the beaded film is given by

$$
A_{\text {eff }}=N A_{o}
$$

where $N$ is the number of $\mathrm{Ag}$ particles and $A_{o}$ is the surface of one (spherical) particle:

$$
A_{o}=4 \pi r_{o}^{2} / 2
$$

Supposing that all the particles have the same radius, $r_{o}$, the volume of the silver in the beads is

$$
V_{A g}=N V_{o}
$$

where $V_{o}$ is the volume of one (semi-spherical) particle:

$$
V_{o}=4 \pi r_{o}{ }^{3} / 6
$$

If, during the formation of the beaded film, some amount of the Ag was lost (eg, by evaporation, diffusion into the substrate, falling out of particles, etc.) then the

$$
V_{A g}=\alpha V_{\text {Ago }}
$$

relation holds with $\alpha<1$.

Using equations (1), (4), and (5), one arrives at:

$$
\alpha A_{h} d=N 4 \pi r_{o}{ }^{3} / 6
$$

from which the number of particles is

$$
N=\alpha A_{h} d /\left(4 \pi r_{o}{ }^{3} / 6\right)
$$

Putting this into equation (2), the effective antibacterial surface (the surface of the silver particles) is

$$
A_{\text {eff }}=3 \alpha d A_{h} / r_{o}
$$

or its relative value

$$
\eta=A_{\text {eff }} / A_{h}=3 \alpha d / r_{o}
$$

The relative coverage measurable in a SEM picture differs from this only by a factor of two (the surface of a semispherical particle is the double of the area of the circle with the same radius, $r_{o}$ )

$$
\eta_{S E M}=(3 / 2) \alpha d / r_{o}
$$

Now, using the numbers obtained in the experimental part: $d \cong 8 \mathrm{~nm}, r_{\mathrm{o}}=29 \mathrm{~nm}$, and $\eta_{\mathrm{SEM}} \cong 0.25$, we get

$$
\eta_{S E M}=0,42 \alpha, \text { ie }, \alpha \cong 0.6 .
$$

It is important to note that the $d / r_{o}$ ratio, which is the key factor in optimizing the effectiveness of the antibacterial effect, cannot be controlled by simple geometrical manipulations. It is well-known that the radius of the particles formed during beading of a film of $d$ thickness (ie, the value of $\eta$ ) depends on the temperature and time of the heat treatment, on the initial grain size of the film, on the evaporation etc. ${ }^{38}$ In addition, the falling out factor, $\alpha$, can also be dependent on the experimental conditions and also related to the strength of the adhesion. Thus, a further improvement of resistance against ultrasound treatment and the increase of the relative coverage is a challenge for further investigations. 


\section{Conclusion}

The investigation proved our hypothesis that the technology used in this study can produce a titanium surface with anchored silver nanoparticles with significant antibacterial effect that can help to prevent the development of periimplantitis if created on the surface of a dental implant. Further investigations are planned to check for cytotoxicity of the nanoparticle layer and to optimize the surface configuration to achieve higher antibacterial rate if possible. It may also result in a more cost-effective production of antibacterial surface by achieving a higher antibacterial rate by increasing $\eta$ value with a smaller amount of silver to be applied.

\section{Abbreviation list}

Ag-Np, Silver Nanoparticle; ECRIS, Electron Cyclotron Resonance Ion Source; PVD, Physical Vapor Deposition; ECR, Electron Cyclotron Resonance; SRIM, Stopping and Range of Ions in Matter; SNMS, Secondary Neutral Mass Spectrometry; EDS, Energy Dispersive X-ray Spectrometry; CFU, colony forming units; BHI, brain heart infusion broth; ROS, reactive oxygen species; DPSC, dental pulp stem cell.

\section{Ethics statement}

The human dental pulp tissue collection performed in this study was approved by the Ethical Committee of the Hungarian Medical Research Council (approval number: ETT TUKEB 49849-3/2016/EKU). For the use of tissue samples, written information consent was obtained from all human subjects who participated in the investigation.

\section{Acknowledgments}

The research was financed by the Higher Education Institutional Excellence Program of the Ministry of Human Capacities in Hungary, within the framework of the Biotechnology thematic programme of the University of Debrecen, and supported by the GINOP-2.3.2-15-201600011 and GINOP-2.3.2-15-2016-00022 projects. The projects are co-financed by the European Union and the European Regional Development Fund.

\section{Disclosure}

The authors report no conflicts of interest in this work.

\section{References}

1. Derks J, Tomasi C. Peri-implant health and disease. A systematic review of current epidemiology. J Clin Periodontol. 2015;42 (suppl16):S158-S171. doi:10.1111/jcpe.12334
2. Wang G, Jin W, Qasim AM, et al. Antibacterial effect of titanium embedded with silver nanoparticles based on electron-transferinduced reactive oxygen species. Biomaterials. 2017;124:25-34. doi:10.1016/j.biomaterials.2017.01.028

3. Li H, Cui Q, Feng B, Wang J, Lu X, Weng J. Antibacterial activity of $\mathrm{TiO}_{2}$ nanotubes: influence of crystal phase, morphology and $\mathrm{Ag}$ deposition. Appl Surf Sci. 2013;284:179-183. doi:10.1016/j. apsusc.2013.07.076

4. Joya YF, Liu Z, Joya KS, Wang T. Preparation and antibacterial properties of laser-generated silver-anatase nanocomposite film against Escherichia coli and Staphylococcus aureus. Nanotechnology. 2012;23(49):495708. doi:10.1088/0957-4484/23/ $49 / 495708$

5. Hajkova P, Spatenka P, Krumeich J, et al. Antibacterial effect of silver modified $\mathrm{TiO}_{2} / \mathrm{PECVD}$ films. Eur Phys $J$ D. 2009;54:189-193. doi:10.1140/epjd/e2009-00087-7

6. Tallósy SP, Janovák L, Ménesi J, et al. Investigation of the antibacterial effects of silver-modified $\mathrm{TiO} 2$ and $\mathrm{ZnO}$ plasmonic photocatalysts embedded in polymer thin films. Environ Sci Pollut Res Int. 2014;21(19):11155-11167. Epub 2014 Feb 5. doi:10.1007/s11356014-2568-6

7. Uhm SH, Song DH, Kwon JS, et al. E-beam fabrication of antibacterial silver nanoparticles on diameter-controlled $\mathrm{TiO} 2$ nanotubes for bio-implants. Surf Coat Technol. 2013;228:360-366. doi:10.1016/j. surfcoat.2012.05.102

8. Csarnovics I, Hajdu P, Biri S, et al. Preliminary studies of the creation of gold nanoparticles on titanium surface towards biomedical applications. Vacuum. 2016;128:55-58. doi:10.1016/j.vacuum.2016.01.013

9. Shi J, Sun X, Lin Y, et al. Endothelial cell injury and dysfunction induced by silver nanoparticles through oxidative stress via IKK/ NK-kB pathways. Biomaterials. 2014;35(24):6657-6666. doi:10.1016/j.biomaterials.2014.04.093

10. Pacurari M, Yin XJ, Zhao J, et al. Raw single-wall carbon nanotubes induce oxidative stress and activate MAPKs, AP-1, NF-kB, and Akt in normal and malignant human mesothelial cells. Environ Health Perspect. 2008;116(9):1211-1217. doi:10.1289/ehp.10924

11. Rahman M, Wang J, Patterson T, et al. Expression of genes related to oxidative stress in the mouse brain after exposure to silver-5 nanoparticles. Toxicol Lett. 2009;187:15-21. doi:10.1016/j. toxlet.2009.01.020

12. Doudi M, Setorki M. Acute effect of nanosilver to function and tissue liver of rat after intraperitioneal injection. J Biol Sci. 2014;14 (3):213-219. doi:10.3923/jbs.2014.213.219

13. Inkielewicz-Stepniak I, Santos-Martinez MJ, Medina C, Radomski MW. Pharmacological and toxicological effects of co-exposure of human gingival fibroblasts to silver nanoparticles and sodium fluoride. Int $J$ Nanomedicine. 2014;9:1677-1687. doi:10.2147/IJN.S59172

14. Ziegler JF, Ziegler MD, Biersack JP. SRIM - the stopping and range of ions in matter (2010). Nucl Instrum Methods Phys Res Sect B. 2010;268:1818-1823. doi:10.1016/j.nimb.2010.02.091

15. Vad K, Csik A, Langer GA. Secondary neutral mass spectrometry a powerful technique for quantitative elemental and depth profiling analyses of nanostructures. Spectrosc Eur. 2009;21:13-17.

16. Bonyár A, Csarnovics I, Veres M, et al. Investigation of the performance of thermally generated $\mathrm{Au} / \mathrm{Ag}$ nanoislands for SERS and LSPR applications. Procedia Eng. 2016;168:1152-1155. doi:10.1016/j.proeng.2016.11.384

17. Biri S, Rácz R, Pálinkás J. Status and special features of the Atomki ECR ion source. Rev Sci Instrum. 2012;83(2):02A341. doi:10.1063/1.3673006

18. Rácz R, Biri S, Hajdu P, et al. Application of an ECR ion source for ionic functionalization of implant materials on the nanoscale. $21 \mathrm{st}$ International Workshop on ECR Ion Sources.24-28 August 2014. Nizhny Novgorod, Russia: ECRIS; 2014.

19. ISO22196:2007(E) Measurement of antibacterial activity on plastics and other non-porous surfaces. 
20. Zhu W, Zhang Z, Gu B, Sun J, Zhu L. Biological activity and antibacterial property of nano-structured $\mathrm{TiO} 2$ coating incorporated with $\mathrm{Cu}$ prepared by micro-arc oxidation. J Mater Sci Technol. 2013;29(3):237-244. doi:10.1016/j.jmst.2012.12.015

21. Zheng Y, Li J, Liu X, Sun J. Antimicrobial and osteogenic effect of Ag-implanted titanium with a nanostructured surface. Int $J$ Nanomedicine. 2012;7:875-884. Epub 2012 Feb 21. doi:10.2147/IJN.S28450

22. Herigstad B, Hamilton M, Heersink J. How to optimize the drop plate method for enumerating bacteria. J Microbiol Methods. 2001;44 (2):121-129.

23. Lindhe J, Meyle J, working group D of the 6th EWOP (2008). Peri implant deseases: consensus report of the Sixth European workshop on periodontology. J Clin Periodontol. 2008;35(suppl18):282-285. doi:10.1111/j.1600-051X.2008.01283.x

24. Neugebauer J, Nickenig HJ, Zöller JE, Rothamel D. Guideline: peri-implant inflammation: prevention-diagnosis-therapy. 10th European Consensus Conference (EuCC) 2015 in Cologne. Eur J Dent Implantol. 2015;11(2):37-44.

25. Noronha VT, Paula AJ, Durán G, et al. Silver nanoparticles in dentistry. Dent Mater. 2017;33:1110-1126. doi:10.1016/j.dental.2017.07.002

26. Klueh U, Wagner V, Kelly S, Johnson A, Bryers JD. Efficacy of silver-coated fabric to prevent bacterial colonization and subsequent device-based biofilm formation. J Biomed Mater Res. 2000;53 (6):621-631

27. Zare Y, Shabani I. Polymer/metal nanocomposites for biomedical application. Mater Sci Eng C. 2016;60:195-203. doi:10.1016/j. msec.2015.11.023

28. Akhavan O, Ghaderi E. Toxicity of graphene and graphene oxide nanowalls against bacteria. ACS Nano. 2010;4:5731-5736. doi:10.1021/nn101390x

29. Prakash J, Kumar P, Harris RA, et al. Synthesis, characterization and multifunctional properties of plasmonic Ag-TiO2 nanocomposites. Nanotechnology. 2016;27(35):355707-355726. doi:10.1088/0957$4484 / 27 / 35 / 355707$
30. Liu C, Geng L, Yu Y, Zhang Y, Zhao B, Zhao Q. Mechanisms of the enhanced antibacterial effect of $\mathrm{Ag}-\mathrm{TiO}_{2}$ coatings. Biofouling. 2018;34(2):190-199. doi:10.1080/08927014.2017.1423287

31. Mao C, Xiang Y, Liu X, et al. Photo-inspired antibacterial activity and wound healing acceleration by hydrogel embedded with Ag/ $\mathrm{Ag} @ \mathrm{AgCl} / \mathrm{ZnO}$ nanostructures. ACS Nano. 2017;11(9):9010-9023. doi:10.1021/acsnano.7b03513

32. Jin C, Liu X, Tan L, et al. Ag/AgBr loaded mesoporous silica for rapid sterilization and promotion of wound healing. Biomater Sci. 2018;6(7):1735-1744. doi:10.1039/c8bn00353j

33. Choi J, Wang NS. Nanoparticles in biomedical applications and their safety concerns. In Fazel R, editor. Biomedical Engineering - from Theory to Applications. InTechOpen; 2011. [Published on August 29, 2011]. Available from:. https://www.intechopen.com/books/biomedi cal-engineering-from-theory-to-applications/nanoparticles-inbiomedical-applications-and-their-safety-concerns. doi:

34. Chaloupka K, Malam Y, Seifalian AM. Nanosilver as a new generation of nanoproduct in biomedical applications. Trends Biotechnol. 2010;28:580-588. doi:10.1016/j.tibtech.2010.07.006

35. Kang KA, Jubg HY, Lym JS. Cell death by polyvinylpyrolidine-coated silver nanoparticles is mediated by ROS dependent signalling. Biomol Ther. 2012;20:399-405. doi:10.4062/biomolther.2012.20.4.399

36. Foldbjerg R, Dang DA, Autrup H. Cytotoxicity and genotoxicity of silver nanoparticles in the human lung cancer line, A549. Arch Toxicol. 2011;85(7):743-750. doi:10.1007/s00204-010-0545-5

37. Cao H, Liu X, Meng F, Chu PK. Biological actions of silver nanoparticles embedded in titanium controlled by micro-galvanic effects. Biomaterials. 2011;32(3):693-705. doi:10.1016/j.biomaterials.2010.09.066

38. Beszeda I, Imre AW, Gontier-Moya EG, Moya F, Beke DL, Si Ahmed A. Kinetics of morphological changes in nanoscale metallic films followed by auger electron spectroscopy. Defect Diffus Forum. 2003;216-217:269-274. doi:10.4028/www.scientific.net/DDF.216217.269
International Journal of Nanomedicine

\section{Publish your work in this journal}

The International Journal of Nanomedicine is an international, peerreviewed journal focusing on the application of nanotechnology in diagnostics, therapeutics, and drug delivery systems throughout the biomedical field. This journal is indexed on PubMed Central, MedLine, CAS, SciSearch ${ }^{\mathbb{}}$, Current Contents ${ }^{\mathbb{R}} /$ Clinical Medicine,
Journal Citation Reports/Science Edition, EMBase, Scopus and the Elsevier Bibliographic databases. The manuscript management system is completely online and includes a very quick and fair peer-review system, which is all easy to use. Visit http://www.dovepress.com/ testimonials.php to read real quotes from published authors. 